\title{
Finitely generated cyclic extensions of free groups are residually finite
}

\section{Gilbert Baumslag}

\begin{abstract}
We establish the result that a finitely generated cyclic extension of a free group is residually finite. This is done, in part, by making use of the fact that a finitely generated module over a principal ideal domain is a direct sum of cyclic modules.
\end{abstract}

\section{Introduction}

The purpose of this note, as the title suggests, is to prove the following

THEOREM. A finitely generated cyclic extension of a free group is residually finite.

There are a host of finitely generated groups with a single defining relation to which this theorem applies. These groups include the fundamental groups of two-dimensional surfaces (both orientable and non-orientable) as well as the groups

$$
\operatorname{sp}\left(a, b, c: c^{n}=[a, b]\right) \quad(n=1 ; 2, \ldots) .
$$

The residual finiteness of these groups is well-known (see for example, [1], [2], [4] and [5]). However the proof of the theorem provides essentially. new information, even about these groups. An explicit example of a one-relator group which was not known to be residually finite until

Received 18 January 1971. It is a pleasure for the author to acknowledge that this work has profited enormously from some conversations with Mary Kennedy Wright. Support from the National Science Foundation is also gratefully acknowledged. 
now is the group

$$
G=\operatorname{sp}\left(a, b, c:\left[a^{2}, b\right]\left[c, a^{-1} b a\right]\right) .
$$

It is not difficult to show that a finitely generated group $G$ which is an infinite cyclic extension of a free group is not always a one-relator group. So our theorem has meaning apart from groups with a single defining relation, even in this case.

\section{Some useful lemmas}

The proof of the theorem depends in large measure on the following lemmas.

LEMMA 1. A finitely generated module over a principal ideal domain $R$ is a direct sum of a finite number of cyclic modules.

Lemma $I$ is a celebrated classical theorem (see for example [8], p. 86 for a proof). We shall apply it in the case where $R$ is the group algebra of an infinite cyclic group over a field of $p$ elements ( $p$ a prime).

The second result we shall need (and which can be proved directly without too much difficulty ( $c f$. for example [3])) is

LEMMA 2. Let $\underline{\mathrm{V}}$ be a nilpotent variety of prime exponent.

Furthexmore let $F$ be a free group in $\underline{\underline{V}}$. Then any set of elements of $F$ which are independent modulo the derived group $F^{\prime}$ of $F$ freely generate a free group in $\underline{\underline{V}}$.

Finally we shall make use of

LEMMA 3. Let $F$ be a free group and let $f \in F(f \neq 1)$. Then there exists a nilpotent variety $\underline{\underline{V}}$ of prime exponent such that $f \notin V(F)$ (where, as usual, $V(F)$ is the unique minimal normal subgroup of $F$ satisfying $F / V(F) \in \underline{\underline{\mathrm{V}}})$.

The proof of Lemma 3 is a consequence of two theorems, one due to Magnus [9], the other due to Higman [7]. More illuminatingly, denoting the $n$-th term of the lower central series of $F$ by $\lambda_{n} F$, there exists an integer $n$ such that $f \neq \lambda_{n} F$ (Magnus [9]). Moreover $F / \lambda_{n} F$ is torsion-free (Magnus [9]). Now the subgroups of prime index in a finitely generated torsion-free nilpotent group have trivial intersection (Higman 
[7]). It follows that there is a nilpotent variety $\underline{V}$ of prime exponent such that

$$
f \lambda_{n} F \notin V\left(F / \lambda_{n} F\right)
$$

Hence

$$
f \notin V(F)
$$

as desired.

\section{A crucial proposition}

The main (indeed essentially the only) step in the proof of the theorem is the proof of the following proposition.

PROPOSITION. Let $G$ be a finitely generated group with a normal subgroup $N$ such that $G / N$ is infinite cyclic. If $N$ is free in $\alpha$ nilpotent variety $\underline{\mathrm{V}}$ of prime exponent $p$ then $G$ is residually finite.

Proof. We make use of Lemma 1 and Lemma 2 to describe this extension $G$ of $N$ by an infinite cyclic group in sufficiently concise terms so as to be able to deduce the residual finiteness of $G$.

To this end we begin by choosing $t \in G$ so that

$$
G=\operatorname{SP}(N, t) \text {. }
$$

Since $G / N$ is infinite cyclic, $G$ is a split extension of $N$ by $g p(t)$ :

$$
G=N_{\mathrm{gP}}(t) \text { and } N \cap \mathrm{gP}(t)=1 \text {. }
$$

We put

$$
M=N / N^{\prime}
$$

Let us denote the group algebra of $g p(t)$ over the field of $p$ elements by $R$. Then, writing $M$ additively, we have $p x=0$ for every $x \in M$. So $M$ may be regarded as an $R$-module once the action of $t$ on $M$ is defined (by conjugation):

$$
a N^{\prime} \cdot t=t^{-1} a t N^{\prime} \quad(a \in N) .
$$

Now $G$ is finitely generated. It follows that the $R$-module $M$ is finitely generated. Hence, by Lemma $1, M$ is the direct sum of a finite number of cyclic modules: 


$$
M=M_{1} \oplus \ldots \oplus M_{k} \oplus M_{k+1} \oplus \ldots \oplus M_{l} \quad(z \geq k) .
$$

Our notation here has been chosen in such a way that $M_{1}, \ldots, M_{k}$ are free whereas $M_{k+1}, \ldots, M_{l}$ are all torsion-modules.

Let $\varepsilon_{i}$ be a generator of $M_{i}$ for each $i=1,2, \ldots, l$. Furthermore let us denote the set of all integers by $Z$. Now put

$$
\varepsilon_{i, j}=\varepsilon_{i} \cdot t^{j} \quad(j \in Z, i=1,2, \ldots, k) \text {. }
$$

Since $M_{i}$ is free $(i=1,2, \ldots, k)$ it follows that the elements

$$
\ldots, \varepsilon_{i,-1}, \varepsilon_{i, 0}, \varepsilon_{i, 1}, \ldots
$$

are a basis for $M_{i}$, where here we regard $M_{i}$ as a vector space over the field of $p$ elements.

Consider now the submodules $M_{k+1}, \ldots, M_{\eta}$. We choose positive integers $n_{k+1}, \ldots, n_{l}$ so that the elements

$$
\varepsilon_{i, 0}, \varepsilon_{i, 1}, \ldots, \varepsilon_{i, n_{i}-1}(i=k+1, \ldots, l)
$$

constitute a basis for the vector space $M_{i}$ where here

$$
\varepsilon_{i, j}=\varepsilon_{i} \cdot t^{j}
$$

It follows that, for $i=k+1, \ldots, l$,

$$
\varepsilon_{i, n_{i}-1} \cdot t=m_{i, 0} \varepsilon_{i, 0}+\ldots+m_{i, n_{i}-1} \varepsilon_{i, n_{i}-1}
$$

where here $0<m_{i, 0}<p$.

This information can be re-expressed directly in terms of $N$ and $t$. To this end let us choose $e_{i} \in N$ so that

$$
\varepsilon_{i}=e_{i} N^{\prime}
$$

Now, putting

$$
e_{i, j}=t^{-j} e_{i} t^{j}
$$


it follows from the comments above that the following statements hold.

(i) The elements

$$
\ldots, e_{i,-1}, e_{i, 0}, e_{i, 1}, \cdots
$$

are linearly independent modulo $N^{\prime}$ for $i=1,2, \ldots, k$, and similarly so too are the elements

$$
e_{i, 0}, e_{i, 1}, \cdots, e_{i, n_{i}-1}
$$

for $i=k+1, \ldots, i$.

(ii) The set $E$ of all elements given by (2) and (3) generate $N$ modulo $N^{\prime}$ and hence, remembering $N$ is nilpotent, these elements actually generate $N$ itself.

(iii) Since $E$ is comprised of elements which are linearly independent moduio $N^{\prime}, E$ freely generates $N$ (Lemma 2).

(iv) For each $i$ satisfying $k+l \leq i \leq l$ we have

$$
t^{-1} e_{i, n_{i}-1} t=e_{i, 0}^{m, 0} \ldots e_{i, n_{i}-1}^{m_{i, n_{i}-1}} f_{i} \quad\left(f_{i} \in N^{\prime}\right)
$$

where the $m_{i, j}$ are those given by (1).

This information given by (i), (ii), (iii) and (iv) is sufficient for us to be able to deduce that $G$ is residually finite. Thus suppose $g \in G$ $(g \neq 1)$. Our objective is to find a homomorphism $\varphi$ of $G$ into a finite group such that $g \varphi \neq 1$. If $g \neq N$ the existence of such a homomorphism is easily verified. Thus for the remainder of the proof of the proposition we shall assume that $g \in N$. We shall choose a homomorphic image $\tilde{G}$ of $G$ so that there is a homomorphism of $G$ to $\tilde{G}$ of the desired kind.

We repeat that $g \in N$. Since $E$ generates $N$ (see (ii)) there exists a positive integer $n$ such that

$$
\begin{array}{r}
\tilde{N}=\operatorname{sp}\left(e_{1,-n}, \ldots, e_{1,0}, \ldots, e_{1, n} ; \ldots ; e_{k,-n}, \ldots, e_{k, 0}, \ldots, e_{k, n} ;\right. \\
\left.e_{k+1,0}, \ldots, e_{k+1, n_{k+1}-1} ; \ldots ; e_{\imath, 0}, \ldots, e_{\imath, n_{2}-1}\right)
\end{array}
$$

contains all the elements 


$$
g, f_{k+1}, \cdots, f_{\imath}
$$

It follows from (iii) that $\tilde{N}$ is a free group in $\underline{\underline{V}}$ freely generated by the elements exhibited.

Our next move is to define an automorphism $\tau$ of $\tilde{N}$ which mimics the action of $t$ on $N$. The effect of $\tau$ on the generators of $\tilde{N}$-is defined by

$$
\begin{aligned}
& e_{1,-n} \tau=e_{1,-n+1}, \ldots, e_{1,0} \tau=e_{1,1}, \ldots, e_{1, n} \tau=e_{1,-n} \\
& e_{k,-n} \tau=e_{k,-n+1}, \ldots, e_{k, 0} \tau=e_{k, 1}, \ldots, e_{k, n} \tau=e_{k,-n} \\
& e_{k+1,0} \tau=e_{k+1,1}, \ldots, e_{k+1, n_{k+1}-1}^{\tau}=e_{k+1,0}^{m_{k+1,0}} \ldots e_{k+1, n_{k+1}-1}^{m_{k+1, n_{k+1}} f_{k+1}}
\end{aligned}
$$

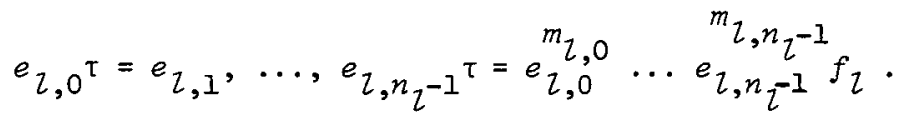

To see that $\tau$ does indeed define an automorphism, observe that the images of the given free generators of $\tilde{N}$ generate $\tilde{N}$ modulo $\tilde{N}^{\prime}$. Hence they generate $\tilde{N}$ since $\tilde{N}$ is nilpotent. But $\tilde{N}$ is finite. So $\tau$ is an automorphism of $\tilde{N}$ (of finite order). Let $\tau$ be of order $r$ and let $\operatorname{gp}(Z)$ be a cyclic group of order $r$ generated by $Z$. Finally let $\tilde{G}$ be the split extension of $\tilde{N}$ by $g p(Z)$ with $Z$ inducing the automorphism $\tau$ of $\tilde{N}$ :

$$
\tilde{G}=\operatorname{sp}\left(\tilde{N}, Z ; Z^{-1} u Z=u \tau \quad(u \in \tilde{N})\right) .
$$

The group $\tilde{G}$ is clearly finite.

There is a natural homomorphism $\varphi$ of $G$ onto $\tilde{G}$ defined by

$$
\varphi: t+z, e_{i, 0} \rightarrow e_{i, 0}(1 \leq i \leq l) .
$$

To see that this mapping does define a homomorphism of $G$ onto $\tilde{G}$ it is enough to observe that the relations in $G$ between the elements $e_{i, 0}$ and 
$t$ are satisfied by the elements $e_{i, 0}$ and $Z$ - this follows from (2), (3) and (4). Clearly

$$
g \varphi=g .
$$

Hence $g \varphi \neq 1$ and the proof of the proposition is now complete.

\section{Some final remarks}

The proof of the theorem is now an immediate consequence of Lemma 3 and the Proposition once one observes that a finite extension of a residually finite group is residually finite.

It is worth pointing out that a finitely generated cyclic extension of a residually finite group need not be residually finite. Indeed if $G$ is the wreath product of a free group $U$ of rank two by an infinite cyclic group then $G$ is not residually finite (Gruenberg [6], Theorem 3.2). But $G$ is a finitely generated cyclic extension of a direct product $F$ of free groups. Of course $F$ is residually finite, but as we remarked $G$ is not.

\section{References}

[1] Gilbert Baumslag, "On generalised free products", Math. 2. 78 (1962), 423-438.

[2] Gilbert Baumslag, "On the residual finiteness of generalised free products of nilpotent groups", Trans. Amer. Math. Soc. 106 (1963), 193-209.

[3] Gilbert Baumslag, "Some subgroup theorems for free v-groups", Trans. Amer. Math. Soc. 108 (1963), 516-525.

[4] Bruce Chandler, "A representation of a generalized free product in an associative ring", Comm. Pure App 2. Maths. 21 (1968), 271-288.

[5] Karen N. Frederick, "The Hopfian property for a class of fundamental groups", Comm. Pure Appl. Maths. 16 (1963), 1-8.

[6] K.W. Gruenberg, "Residual properties of infinite soluble groups", Proc. London Math. Soc. (3) 7 (1957), 29-62. 
[7] Graham Higman, "A remark on finitely generated nilpotent groups", Proc. Amer. Math. Soc. 6 (1955), 284-285.

[8] Nathan Jacobson, Lectures in abstract algebra, Vol. II (Van Nostrand, New York, Toronto, London, 1953).

[9] Wi the Im Magnus, "Beziehungen zwischen Gruppen und Idealen in einem speziellen Ring", Math. Ann. 111 (1935), 259-280.

Rice University,

Houston,

Texas, USA. 